УДК 004.725.5

\title{
C.B. Сахарова
}

Одесская национальная академия пищевых технологий, институт холода, криотехнологий и экоэнергетики им. В.С. Мартыновского, ул. Дворянская, 1/3, г. Одесса, 65082

\section{АНАЛИЗ И ОБОСНОВАНИЕ ВЫБОРА ПАРАМЕТРОВ СЕТЕЙ ПРОВОДНОГО ДОСТУПА}

Выполненные исследования относятся к области проектирования перспективных сетей доступа. Работа посвящена анализу параметров сетей доступа и выбору наиболее значимых среди них. Приведены результаты исследований для проводных решений организации сети.

Ключевые слова: сеть доступа, параметры сетей доступа.

\section{C.B. Cахарова}

Одеська національна академія харчових технологій, інститут холоду, кріотехнологій і екоенергетики, ім. В.С. Мартиновського, вул. Дворянська, 1/3, м. Одеса, 65082

\section{АНАЛІЗ І ОБГРУНТУВАННЯ ВИБОРУ ПАРАМЕТРІВ МЕРЕЖ ПРОВОДОВОГО ДОСТУПУ}

\begin{abstract}
Виконані дослідження відносяться до галузі проектування перспективних мереж доступу.Робота присвячена аналізу параметрів мереж доступу і вибору найбільш значущзих серед них. Наведено результати досліджень для дротяних рішень організації мережі.
\end{abstract}

Ключові слова: мережа доступу, параметри мереж доступу.

\section{S.V. Sakharova}

Odessa national academy of food technologies, Institute of the cold, cryotechnologies and ecoenergetics, 1/3 Dvoryanskaya str., Odessa, 65082

\section{ANALYSIS AND JUSTIFICATION OF NETWORK WIRELINE ACCESS PARAMETERS SELECTION}

The executed researches belong to the area of perspective access networks design. The work is devoted to the access networks parameters analysis and to the choice of the most significant among them. Results of researches for wire decisions of the network organization are given.

Keywords: access network, parameters of access networks.

\section{I. ВВЕДЕНИЕ}

Развитие телекоммуникаций приводит к появлению новых инфокоммуникационных услуг (ИКУ) и одновременно усложняется проблема доступа к ним. При введении каждой новой услуги возможно изменение требований к сети и сетевому оборудованию в связи с необходимостью обеспечения пропускной способности, видов трафика и процедур обслуживания, специфичных именно для этой услуги.

Тема исследования связана с одной из основных проблем в области телекоммуникаций, которой является создание сетей следующего поколения Next Generation Network (NGN), предназначенных для предоставления пользователям всего набора ИКУ. Составляющей $N G N$ являются сети доступа (СД), обеспечивающие пользователям доступ ко всем ИКУ по единой линии доступа (ЛД). Параметры ЛД определяют качество и номенклатуру ИКУ, доступным пользователям NGN. Необходимость создания СД указана в документах международного союза электросвязи (МСЭ) как одна из важнейших задач 21 века [1], а перспекти- вы их развития широко обсуждаются на конференциях и семинарах посвященных развитию инфокоммуникаций. Различные аспекты построения СД освещены в работах Соколова Н.А., Гольдштейна Б.С., Бакланова И.Г., Крендзеля А.В., Хиленко В. В., Михайлова В.Ф., Гайворонской Г.С., Балашова В.А., Зяблова С.В. и других.

Создание СД в настоящее время приобрело особую актуальность, поскольку именно участок доступа является тем сегментом телекоммуникационной сети (ТC), который тормозит внедрение широкополосных высококачественных ИКУ, за счет которых оператор может иметь значительную прибыль. Кроме того, модернизация абонентских сетей и создание на их базе перспективных СД является третьим этапом преобразования ТС, первыми двумя этапами которого является замена аналоговых систем передачи и узлов коммутации на цифровые. Эти этапы осуществляются возрастающими темпами, а сейчас пришло время третьего заключительного этапа, который непосредственно связан с темой работы.

В настоящее время проектирование СД основано на методах расчета абонентских телефонных 
сетей, что недопустимо, поскольку СД отличаются и структурой и функциями, которые они выполняют, а так же набором параметров, поэтому СД должны создаваться на других принципах. Поскольку концепция СД [2] разработана сравнительно недавно, на сегодняшний день не существует апробированных методов их проектирования, поэтому возникла необходимость в разработке последовательности процесса создания СД, а для это необходимо провести анализ параметров СД, влияющих на процесс проектирования.

\section{II. ПОСТАНОВКА ЗАДАЧИ}

В настоящее время применяется огромное разнообразие технологий доступа к ИКУ, существует множество взглядов и подходов к построению СД [3-5]. При создании СД необходимо учесть множество параметров СД и требований, выдвигаемых к ней [6,7]. Поскольку исследуемые СД имеют набор параметров, отличный от параметров существующих абонентских сетей, необходимо провести анализ и составить характеристику этих параметров, выявить корреляцию между ними и привести их в форму, удобную для моделирования. Таким образом, в постановке задачи отмечается необходимость решения задачи анализа, в результате чего определяется перечень параметров, являющихся исходными для создания СД, и тех, которые не существенно влияют на процесс создания сети и учетом которых можно пренебречь. Целью исследования является повышение эффективности проектирования перспективных СД, снижение затрат на их создание и повышение эффективности эксплуатации.

\section{III. СТРУКТУРНЫЙ СОСТАВ СД}

Многими подходами возможно осуществить классификацию, группировку и описание сетей доступа. Однако чаще всего применяется традиционный метод. Он состоит из двух составляющих: текстовое описание и графическое отображение. Особенность текстового описания заключается в том, что такой метод дает возможность читателю в полном объеме ознакомиться с исследуемым объектом. Но текстовое описание не обеспечивает объективного виденья классификационной структуры построения. Поэтому текстовое описание часто дополняется графическим отображением классификационной модели.

Графическое отображение в свою очередь разделяется на две формы представления: в виде табличной структуры и древовидной структуры. Табличная структура чаще всего используется для представления общих классификационных элементов анализируемых объектов. Обычно, табличная структура содержит числовые значения для возможности сравнения исследуемых объектов. Применение данного способа приводит к сжатому представлению.

Древовидная структура обеспечивает наиболее объемно-визуальное представление об объекте исследования. Пошаговое движение по ветвям классификационной модели позволяет проследить и увидеть отличительные особенности в построении объектов на одном уровне дерева.

Так как текстовое описание и графическое отображение взаимодополняют друг друга, то для возможности как можно глубже охарактеризовать СД, в работе задействованы оба метода. Графическая часть работы отображает древовидную структуру классификационной модели построения СД. Для общего ознакомления со структурой модели приведена древовидная схема, отображающая общие элементы классификации для систем проводного доступа. Но здесь остаются скрытыми все тонкости построения для каждого метода реализации СД.

Более глубокое представление об объектах дает разрозненная древовидная структура. В работе она представлена как дополнение к общей классификационной модели построения СД.

При рассмотрении и анализе большого числа существующих решений по реализации СД на данный момент, их можно группировать по следующим признакам: по форме передаваемого сигнала; по назначению сетей доступа; по пропускной способности; по структурному построению; по применяемым технологиям; по управлению; по виду доступа; по классу обслуживаемой местности (рисунок 1).

\section{IV. ГРУППИРОВКА ПАРАМЕТРОВ СД ПО ОБСЛУЖИВАЕМОМУ КЛАССУ МЕСТНО- СТИ}

Для распределения параметров по их важности в процессе создания СД выделяются признаки, по которым можно составить классификацию параметров. Как пример, можно провести классификацию параметров для СД по классу обслуживаемой местности, т.к. при проектировании сети необходимо учесть особенности той местности, для которой она строится.

Учитываются следующие параметры.

1. Географическое положение местности. Территория, на которой планируется построение СД, может располагаться в горной местности, на острове, вблизи объектов, влияющих на функционирование сети и т.д. От этого зависит выбор среды передачи, структуры построения сети.

2. Поверхностная плотность населения - количество жителей на единицу площади. Выделены различия по обслуживаемой плотности населения:

- обслуживающие местность с высокой плотность населения;

- обслуживающие местность со средней плотностью населения;

- обслуживающие местность с низкой плотностью населения.

Высокая поверхностная плотность населения часто наблюдается в центральной части города. Обычно это население многоэтажных домов, рабочие на крупных предприятиях. Для обслужива- 
ния этих районов устанавливаются сети доступа с большой нагрузочной способностью.

Средняя поверхностная плотность населения в основном имеет местность, находящаяся за пределами центральной части города. Это районы города обычно застроены невысокими зданиями (в основном одно - и пятиэтажные постройки). Однако может наблюдаться высокий уровень транспортного движения. Системы сетей доступа обслуживающие эти районы, обычно имеют среднюю нагрузочную способность, вполне достаточную для нормальной работы сети.

Низкая поверхностная плотность населения в основном наблюдается за пределами города, это обычно сельские районы. Для обслуживания этих районов достаточно применить системы доступа с низкой нагрузочной способностью, и большой дальности связи.

3. Также важной характеристикой является поверхностная плотность пользователей - количество пользователей ИКУ на единицу площади.

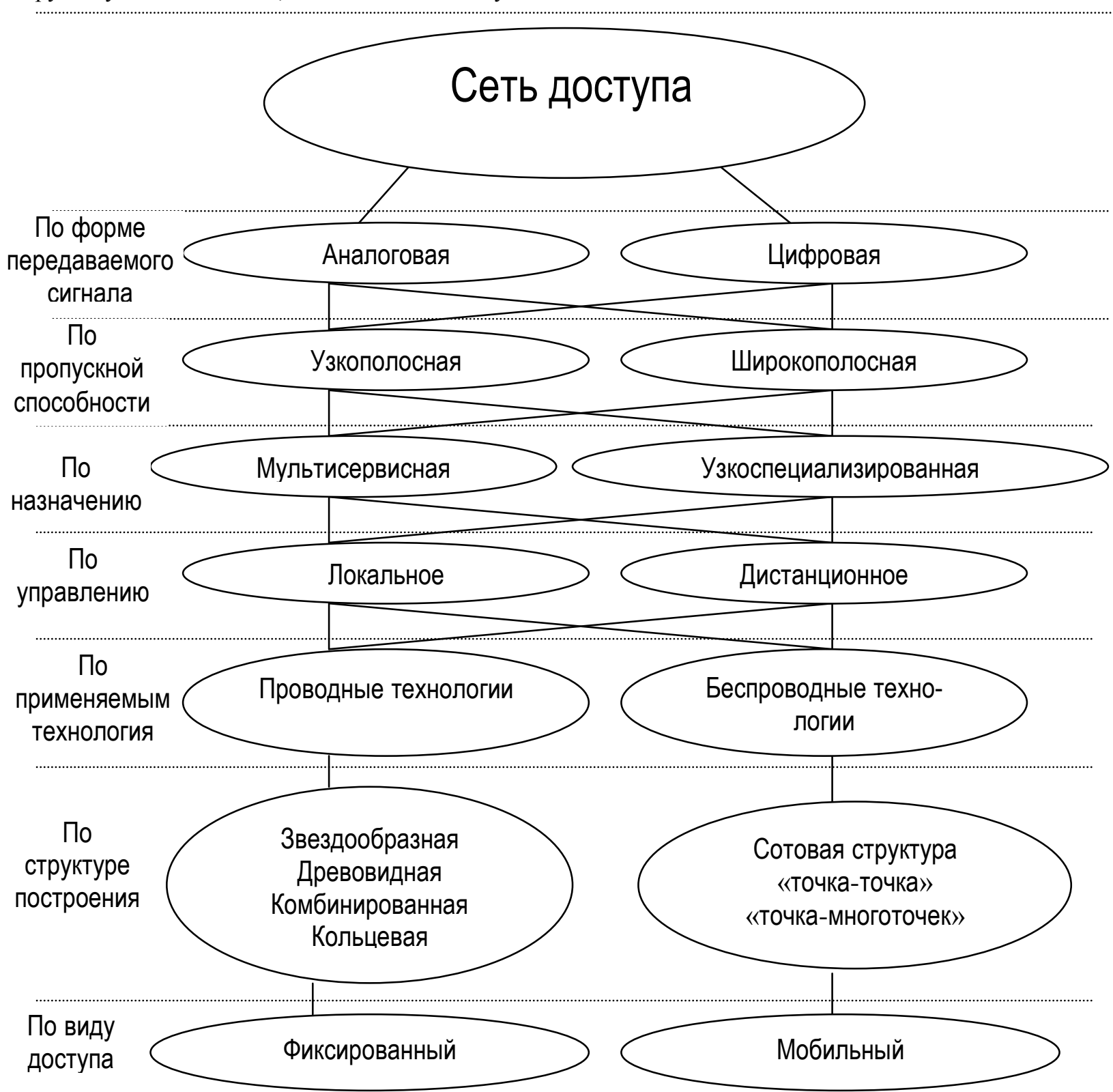

Рисунок 1 -Структурный состав СД

В крупных городах и областных центрах обычно сосредоточенно наибольшее количество пользователей ИКУ, являющихся наиболее требовательными к предоставляемым услугам и к параметрам СД. Сельские районы с доминирующим числом жителей престарелого возраста наоборот отличаются отсутствием большого количества пользователей ИКУ, соответственно нет необходимости вкладывать большие средства в создание СД большой пропускной способности. По параметру поверхностной плотности пользователей, 
местность можно разделить на местности с высокой, средней и низкой плотностью пользователей.

4. Финансовые возможности пользователей. Создание СД должно являться экономически выгодным. Не рационально вкладывать большие денежные средства в создание сети, пользователи которой не имеют финансовой возможности оплачивать весь спектр услуг, предоставляемый данной сетью. Напротив, в районах, жители которых имеют неограниченные финансовые возможности, вероятность обращения к спектру ИКУ с требованием высокой скорости передачи информации повышается. Таким образом, по финансовым возможностям пользователей обслуживаемую местность можно разделить на местность с высокими, средними и низкими финансовыми возможностями пользователей.

5. Также необходимо учитывать тип местности или района - это может быть деловой центр города, спальный район, коттеджный поселок, частный сектор, военный городок, студенческий городок, элитный жилой комплекс, жилой комплекс бизнес класса, дачный сектор, пригородная зона, курортная зона, торговая зона, территория завода и т.д.

Данная группа параметров, не являются непосредственно параметрами СД (параметрами оборудования СД), но имеют существенное влияние на ее структуру, стоимость и процесс эксплуатации.

Tаблица 1 - Группировка параметров проводных СД

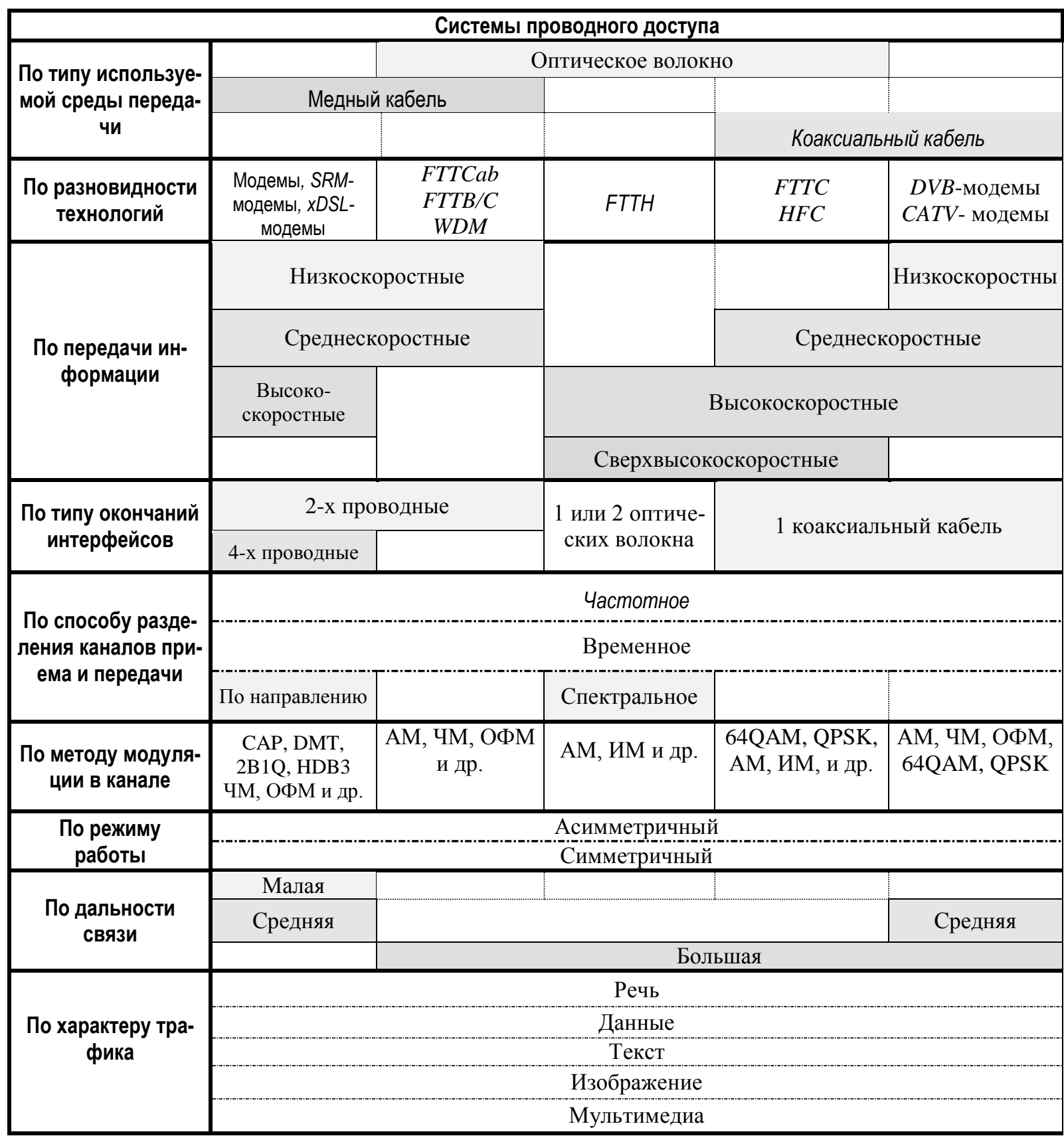




\section{V. ГРУППИРОВКА ПАРАМЕТРОВ ПРОВОД- НЫХ СД}

Организация проводной сети доступа предполагает наличие физической среды для переноса информации, оборудования доступа, систем передачи, а также дополнительных средств, обеспечивающих нормальную работу.

Существующие СД по техническим параметрам группируются по следующим признакам: по типу используемой среды передачи; по разновидностям технологий; по скорости передачи информации; по типу окончания интерфейсов; по способу разделения каналов приёма и передачи; по ме-

Учитывая то, что при рассмотрении систем доступа к ИКУ выделено достаточно большое количество параметров, для удобства выбранные тоду модуляции в канале; по режиму работы; по дальности связи; по характеру трафика и услуг.

Выбранные параметры требуется представить в форме, удобной для моделирования. Весь набор параметров делится на две категории: качественные и количественные параметры, для которых определяются диапазоны и градации принимаемых значений. В постановке задачи исследования ограничимся вопросами создания СД лишь на базе проводных технологий доступа. Пример отображения результатов анализа параметров проводных СД, представленный в виде дерева с использованием программного обеспечения Concept Draw Office MINDMAP, приведен на рис. 2.

- требования пользователей, на которых ориентирована создаваемая СД, такие как скорость передачи информации, дальность связи, коэффи-

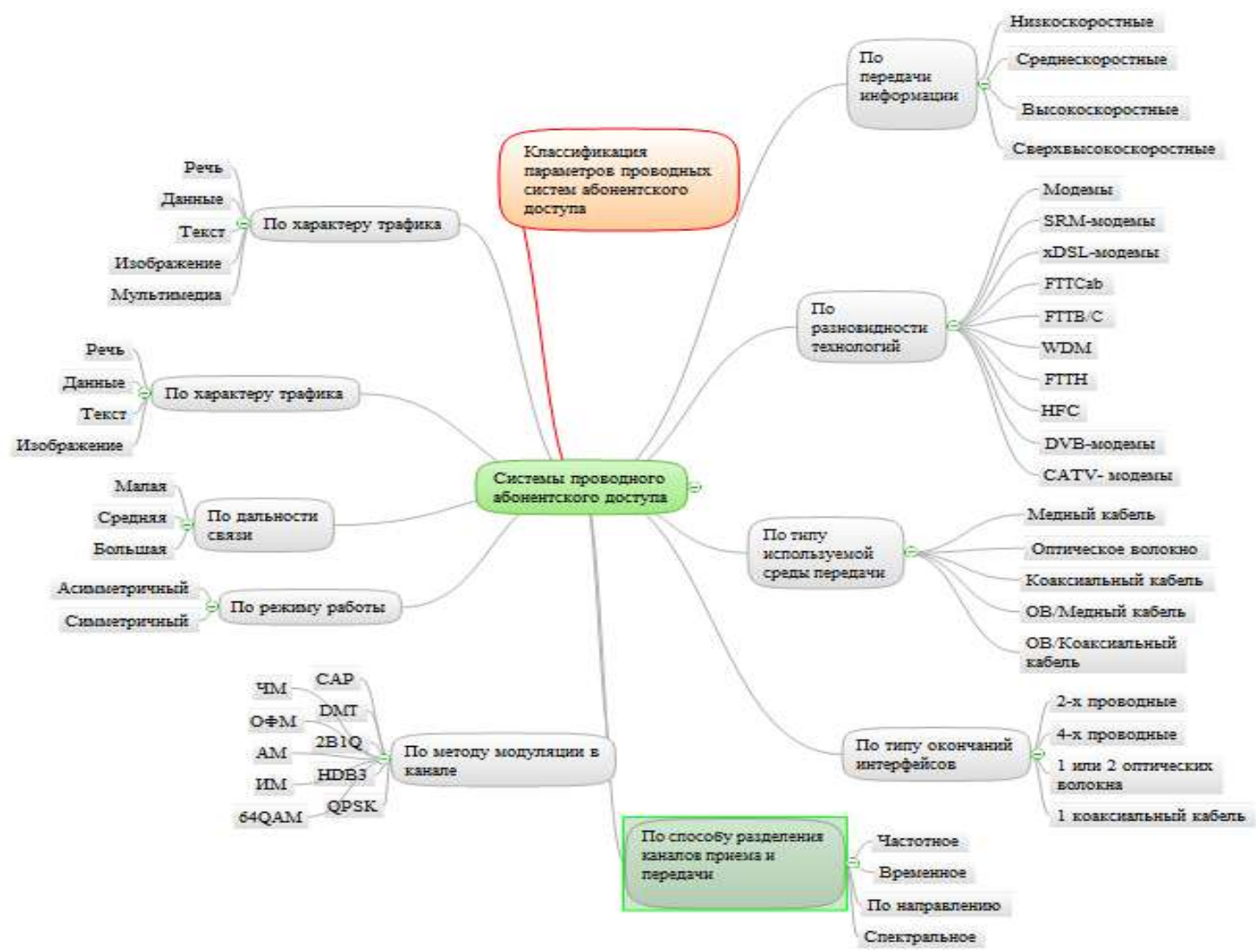

Рисунок 2 - Структура параметров проводных СД

параметры были разделены на группы. Структура исследования, представлена на рисунке 3.

В работе предложен один из способов разделения параметров на группы.

1. Параметры, не являющиеся параметрами СД, но влияющие на процесс моделирования. К ним относятся, например, финансовые возможности пользователей, тип местности, ее географическое положение и т.д.

2. Параметры СД, являющиеся исходными данными для моделирования. Среди них выделяются: циент ошибок, время задержки, вариация задержки и т.П.;

- параметры, основанные на требованиях пользователей, но не являющиеся важными для них, такие как метод модуляции в канале, применяемая технология доступа, метод разделения канала приема и передачи и т.п.

3. В зависимости от набора требований и параметров формируются такие параметры, как стоимость услуг, качество связи. 


\section{Ограничения}

- требования к сети и сетевому оборудованию; качество предоставления услуг.

\section{Заданные значения:}

- стоимость оборудования;

- пропускная способность узлов доступа.

\section{Прогнозируемые параметры:}

- количество пользователей;

- перечень услуг;

- поверхностная плотность и распределение пользователей на территории обслуживания; нагрузка, создаваемая пользователями.

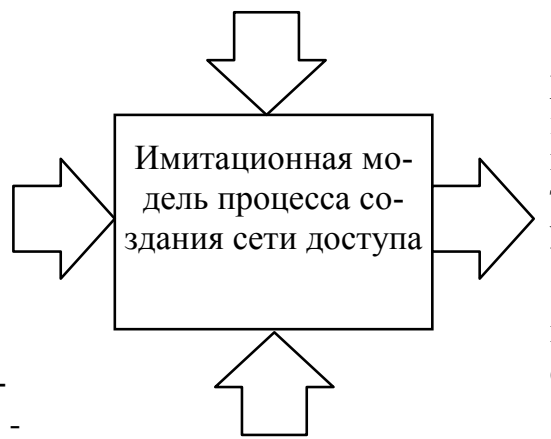

Критерий оптимизации

(стоимость сети, длина линий доступа)
Характеристики сети доступа: количество, места размещения узлов доступа, размеры территорий, обслуживаемых узлами доступа;

длина локального и транспортного сегментов доступу; стоимость сети...

\section{Рисунок 3 - Модель группировки параметров СД}

\section{VI. ВЫВОДЫ}

Анализ исходных параметров сетей доступа позволил выделить те, которые не существенно влияют на процесс создания сети и учетом которых можно пренебречь, и параметры, являющиеся существенными при создании сетей доступа, и влияющие на их структуру и стоимость.

Составлена характеристика каждого из рассматриваемых параметров. Для распределения параметров по их важности в процессе создания сетей доступа выделены признаки, по которым можно составить классификацию параметров.

Весь набор параметров сетей доступа разделен на две категории: качественные и количественные, для которых определены диапазоны и градации принимаемых ими значений. Результаты анализа параметров СД, представлены в табличном виде и в виде дерева с использованием программного обеспечения Concept Draw Office MINDMAP.

Результаты анализа параметров СД представлены автором в работах [8-11].

\section{ЛИТЕРАТУРА}

1. “Международный союз электросвязи (ITU)”, официальное Интернет-представительство, [Электронный pecypc] http://www.itu.int. — Режим доступа: Iwww/ http://www.itu.int / — 01.03.2012 г. — Загл. с экрана.

2. Рекомендация ITU-T G.902 Framework Recommendation on functional access networks (AN) [Электронный pecypc] / ITU: Committed to connecting the world.- Peжим доступа: Iwww/ http://www.itu.int / - 10.03.2012 г. - Загл. с экрана.

3. Гайворонская Г.С. Основные задачи модернизации сетей пользовательского доступа / Г.С Гайворонская., А.И. Котова // Зв’язок. - 2010. - №1 (89). -С. 18-24;
4. Гайворонская Г.С. Концепция пользовательского доступа: Учебник для ВУЗов [Текст] / Г.С Гайворонская - Одесса: ОГАХ, 2008. - 408 с.

5. Соколов Н. А. Сети абонентского доступа [Текст] / Н.А. Соколов - Пермь: ИПК Звезда, 1999. - 154 с.

6. Гайворонская Г. С. Оценка влияния некоторых факторов на процесс развития телекоммуникационных сетей / Г.С Гайворонская // Холодильна техніка і технологія. - 2006. -№2 (100). - С. 95-100.

7. Гайворонская Г. С. Анализ влияния вариации исходных параметров на результаты сетевого планирования / Г.С Гайворонская // Тр. УНИИРТ. - 2006. №3 (47). - С. 102-106;

8. Гайворонская Г.С. Дослідження впливу помилок прогнозу вихідних даних на процес планування мереж доступу / Гайворонська Г.С., Сахарова С.В. // Збірник наукових праць ВІТІ НТУУ „КПІ”. - 2010. - № 2., c 23-29.

9. Сахарова С.В. Задача выбора параметров сети доступа / С.В. Сахарова // Сборник тезисов III МТК «Проблеми телекомунікацій», КПІ, Киев, 21-24 апреля 2009. - С.61.;

10. Гайворонская Г.С. Классификация параметров сетей доступа / Г.С. Гайворонская, С.В. Сахарова // Сборник тезисов Пятой МНТК «Современные информационно-коммуникационные технологии», Крым, Ялта, Ливадия, 05-09 октября 2009. - С.77-78.;

11. Сахарова С.В. Исследование параметров сетей абонентского доступа / С.В. Сахарова // Материалы VIII МНTК «Математическое моделирование и информационные технологии»/ММИТ-2008/ - Одесса: ОГАХ. - 2008.-С. 29.

\section{REFERENCES}

1. "Mezhdunarodnyj sojuz jelektrosvjazi (ITU)", oficial'noe Internet-predstavitel'stvo, [Jelektronnyj resurs] 
http://www.itu.int. - Rezhim dostupa: lwww/ http://www.itu.int / — 01.03.2012 g. — Zagl. s jekrana. 2. Rekomendacija ITU-T G.902 Framework Recommendation on functional access networks (AN) [Jelektronnyj resurs] / ITU: Committed to connecting the world. - Rezhim dostupa: Iwww/ http://www.itu.int / 10.03.2012 g. - Zagl. s jekrana.

3. Gajvoronskaja G.S. Osnovnye zadachi modernizacii setej pol'zovatel'skogo dostupa / G.S Gajvoronskaja., A.I. Kotova // Zv’jazok. - 2010. - №1 (89). -S. 18-24; 4. Gajvoronskaja G.S. Koncepcija pol'zovatel'skogo dostupa: Uchebnik dlja VUZov [Tekst] / G.S Gajvoronskaja - Odessa: OGAH, 2008. - 408 s.

5. Sokolov N. A. Seti abonentskogo dostupa [Tekst] / N.A. Sokolov - Perm': IPK Zvezda, 1999. - 154 s.

6. Gajvoronskaja G. S. Ocenka vlijanija nekotoryh faktorov na process razvitija telekommunikacionnyh setej / G.S Gajvoronskaja // Holodil'na tehnika i tehnologija. 2006. -№2 (100). - S. 95-100.

7. Gajvoronskaja G. S. Analiz vlijanija variacii ishodnyh parametrov na rezul'taty setevogo planirovanija / G.S
Gajvoronskaja // Tr. UNIIRT. - 2006. - №3 (47). S. 102-106;

8. Gajvoronskaja G.S. Doslidzhennja vplivu pomilok prognozu vihidnih danih na proces planuvannja merezh dostupu / Gajvorons'ka G.S., Saharova S.V.// Zbirnik naukovih prac' VITI NTUU „KPI”. - 2010. - № 2, s. 23-29.

9. Saharova S.V. Zadacha vybora parametrov seti dostupa / S.V. Saharova // Sbornik tezisov III MTK «Problemi telekomunikacij», KPI, Kiev, 21-24 aprelja 2009. - C.61.;

10. Gajvoronskaja G.S. Klassifikacija parametrov setej dostupa / G.S. Gajvoronskaja, S.V. Saharova // Sbornik tezisov Pjatoj MNTK «Sovremennye informacionnokommunikacionnye tehnologii», Krym, Jalta, Livadija, 0509 oktjabrja 2009. - C.77-78.;

11. Saharova S.V. Issledovanie parametrov setej abonentskogo dostupa / S.V. Saharova // Materialy VIII MNTK «Matematicheskoe modelirovanie i informacionnye tehnologii» /MMIT-2008/ - Odessa: OGAH. 2008.- C. 29.

Получена в редакции 08.10.2013, принята к печати 03.12.2013 